\title{
Is right iliac fossa mass always points to an appendix or caecum? An incidental rare gynecological diagnosis of Collision Tumour in a Supernumerary ovary
}

\author{
Authors
}

\author{
Dr R. Suganya ${ }^{1}$, Dr S. Priya Banthavi ${ }^{2}$, Dr V.Sarada ${ }^{3}$ \\ ${ }^{1}$ Assistant Professor, Pathology, Trichy SRM Medical College Hospital, Trichy \\ ${ }^{2}$ Associate Professor, Pathology, Trichy SRM Medical College Hospital, Trichy \\ ${ }^{3}$ Professor \& HOD, Pathology, Trichy SRM Medical College Hospital, Trichy
}

\section{Introduction}

A mass in the right iliac fossa with pain is often diagnosed as appendicular mass. The differential diagnosis are ileocaecal tuberculosis, carcinoma caecum, intussusception, tuboovarian mass and rarely lymphoma or ectopic kidney. The existence of supernumerary ovary is very rare and has very few case reports. Supernumerary ovary is an additional ovary which has no connection with either the two normal ovaries or the utero-ovarian ligament, broad ligament or the infundibulo-pelvic ligament. Tumours arising from the supernumerary ovaries are extremely uncommon. One such case report is presented for its unusual diagnosis and rarity.

\section{Case Report}

A 40 year old multigravida was admitted to the Gynaecology outpatient department, Trichy SRM Medical College \& Research centre with a history of abdominal pain for the past one month. Her previous antenatal and postnatal periods were unremarkable. Her medical and surgical histories were uneventful. Ultrasound examination revealed a large cystic mass in the right iliac fossa. The uterus and ovaries were normal. An exploratory laparotomy was proceeded with per operative findings revealing a huge paraovarian mass attached to the left fallopian tube, which was twisted and present in the right iliac fossa. Both ovaries appeared normal. Total abdominal hysterectomy with bilateral salpingooophorectomy along with cystectomy was done and the specimen was sent to Pathology department.

\section{Pathology findings}

Grossing of the specimen revealed uterus with cervix $(9 \times 7 \times 4 \mathrm{~cm})$, left ovary $(4 \times 1.6 \times 0.2 \mathrm{~cm})$, right ovary $(2.5 \times 2 \times 0.5 \mathrm{~cm})$ and a cystic mass attached to the left tubal fimbrial end $(13 \times 10 \times$ $6.5 \mathrm{~cm}$ ). The right fallopian tube measured $3.5 \mathrm{~cm}$ in length and left tube, appeared twisted and measured $7 \mathrm{~cm}$ in length. The cut surface of the uterus, both tubes and ovaries were grossly unremarkable. The cystic mass had no connection with either of the ovaries. The outer surface of the cyst was smooth and congested with intact surface. Cut surface revealed multiple cysts of variable sizes, the largest one measuring $11 \times 10.5$ $\mathrm{cm}$ and contained $20 \mathrm{cc}$ of straw colored fluid. Inner wall of the largest cyst appeared smooth, hemorrhagic with cyst wall thickness ranging from 0.1 to $0.2 \mathrm{~cm}$. The other cysts gave a 


\section{JMSCR Vol||07||Issue||02||Page 289-293||February}

variegated appearance and were filled with mucinous, hemorrhagic and gelatinous substance. One focus revealed ovarian parenchyma with corpus luteum.

Microscopic examination showed disorderly proliferative endometrium with benign adenomatous polyp, chronic cervicitis and unremarkable bilateral tubes and ovaries. Sections from the cystic mass attached to the tube showed ovarian tissue forming parts of cyst wall, with subcortical follicle formation and corpus luteum. The largest cyst was lined by cuboidal epithelium with papillary fronds. Rest of the tumour showed multiple small cystic spaces, one of them showing nodular collection of thyroid follicles containing colloid. Other areas of the wall showed mucinous lining epithelium, cholesterol clefts, siderophages, foci of calcification and condensed fibrous tissue. The diagnosis was made as Supernumerary ovary with collision tumour, the components being serous cystadenoma and mature cystic teratoma.

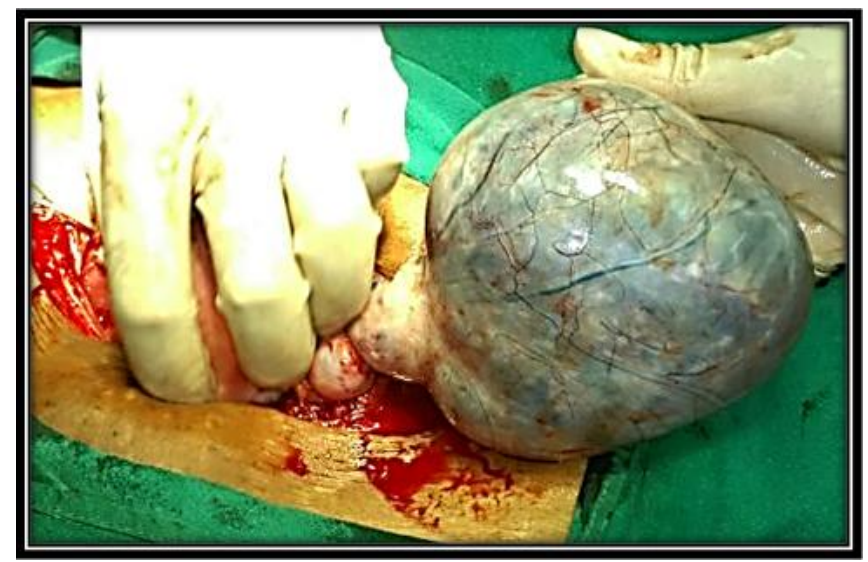

Fig 1: Peroperative findings of the cystic mass

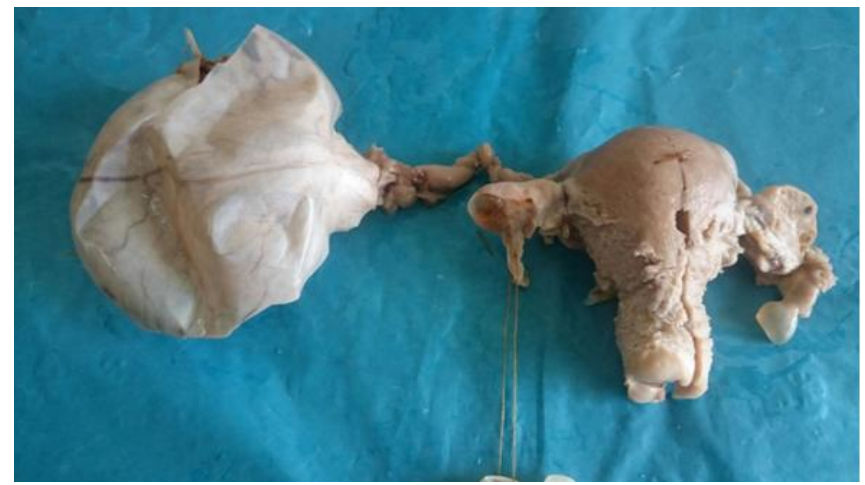

Fig 2: Gross appearance showing both side normal ovaries and a separate cystic mass

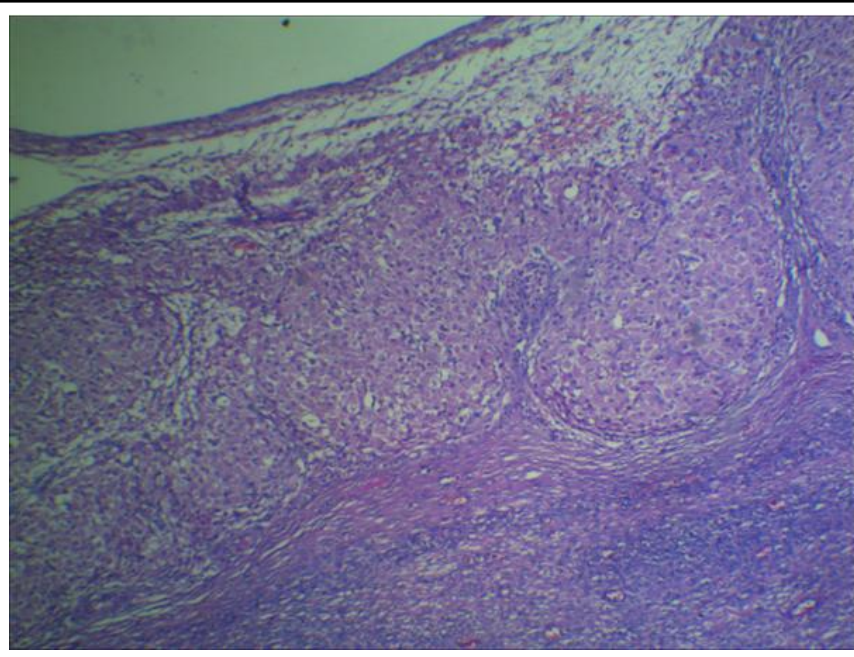

Fig 3: Mass showing normal ovarian parenchyma with corpus luteum (10X, H\&E)

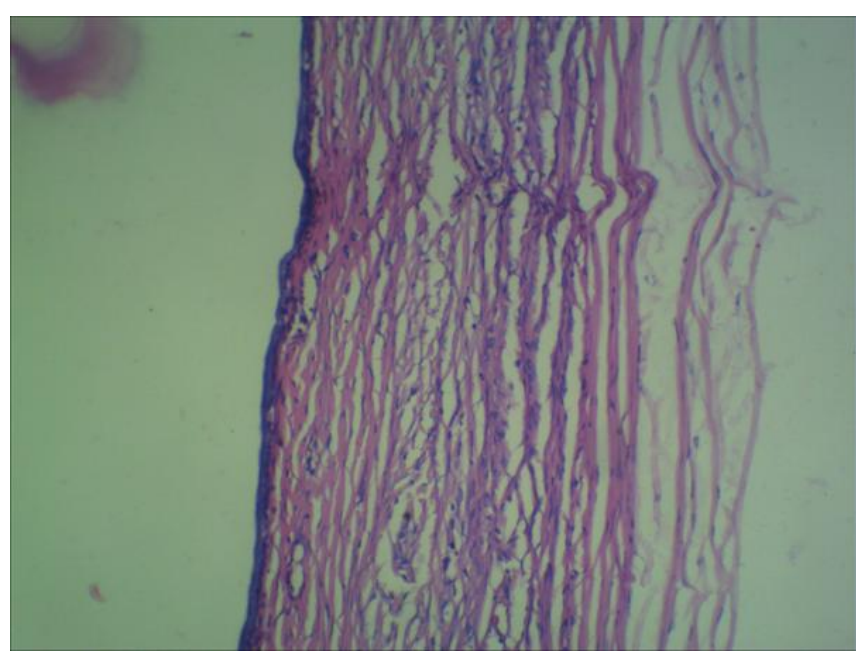

Fig 4: Cyst lined by cuboidal epithelium (serous cystadenoma, 40X, H\&E)

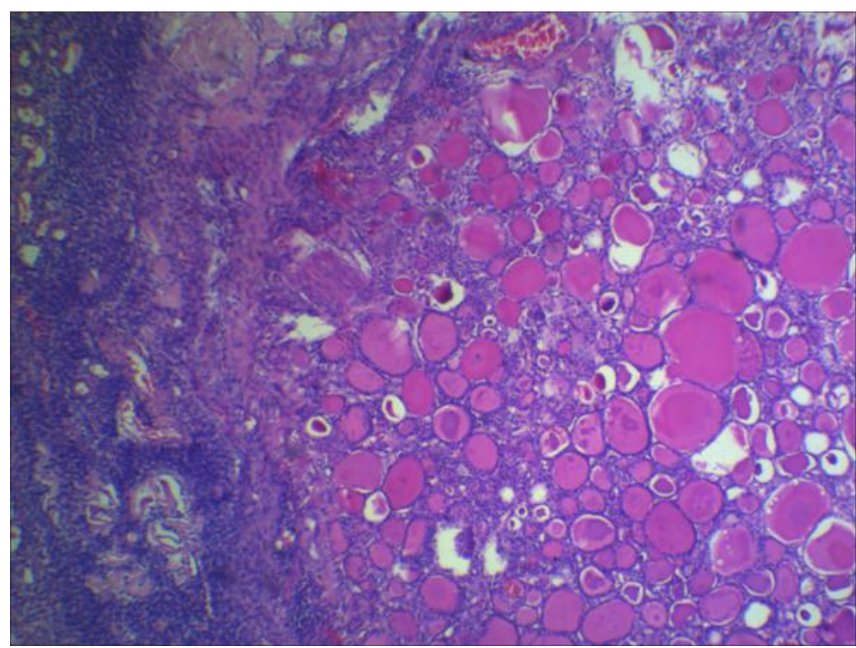

Fig 5: Colloid filled follicles with thyroid parenchyma (10X, H\&E) 


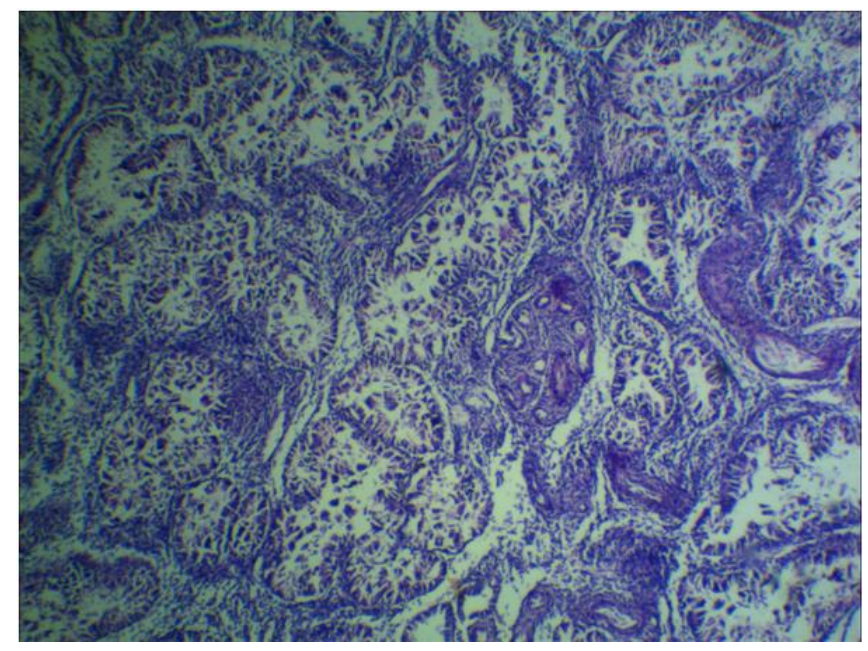

Fig 6: Glandular proliferation (10X, H\&E)

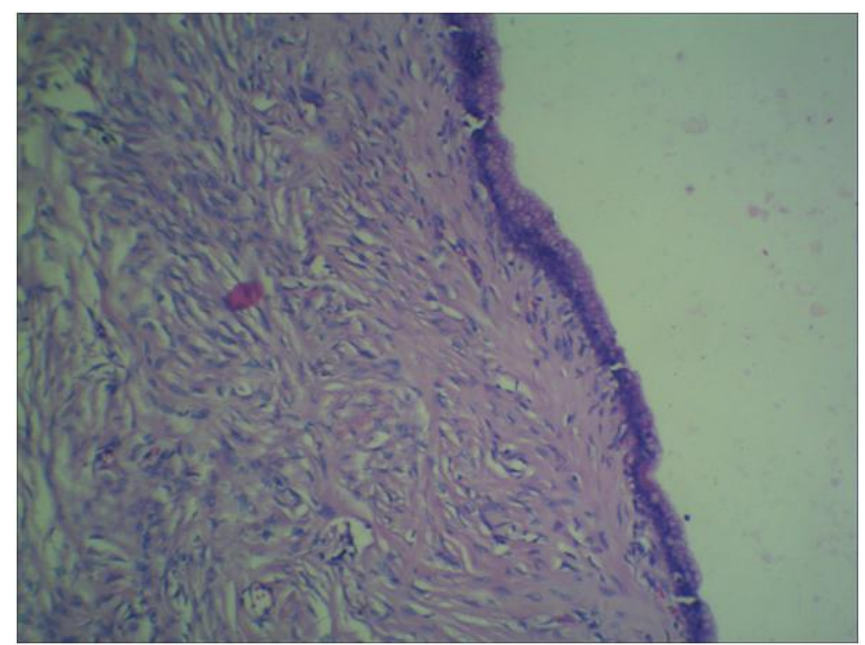

Fig 7: Glands lined by mucinous epithelium (40X, $\mathrm{H} \& \mathrm{E})$

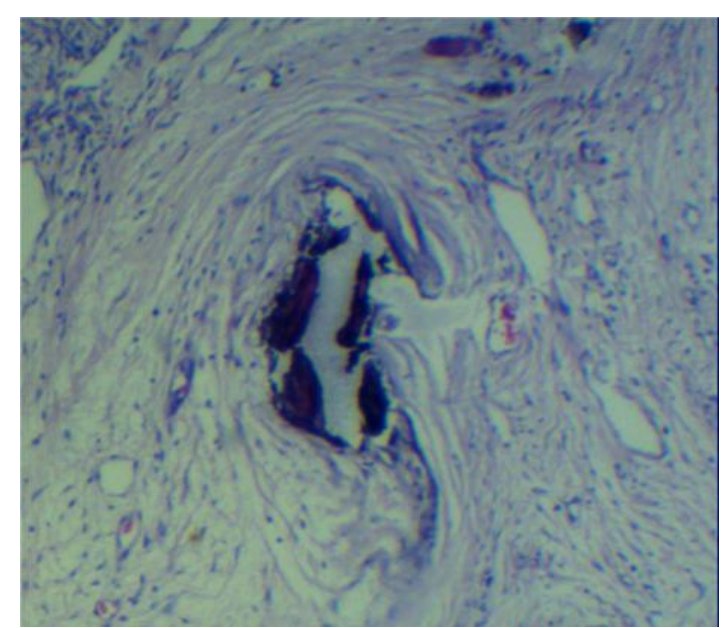

Fig 8: Calcification (40X, H\&E)

\section{Discussion}

The incidence of an additional ovary is very rare being about 1 in 29000 to 1 in 93000. Additional ovary includes supernumerary ovary, accessory ovary and ectopic ovary. These terms are often used interchangeably but are different entities. An ectopic ovary is one in which the ovary is seen in a site other than the normal anatomical position of ovary. Wharton was the first to describe two cases of supernumerary ovary and one accessory ovary in 1959. He classified ectopic ovaries into supernumerary and accessory ovaries based on its relation to the normal ovary. Supernumerary ovary is defined as an ovarian tissue at a different or normal site, but separate from the normal ovary and has no connection with either the ovary or any ligaments like round ligament, broad ligament or utero- ovarian ligament. An accessory ovary is an additional ovary often located near the normal ovary and has a definite connection with it. Embryologically, supernumerary ovary has its origin from a separate primodium and does not share the normal ovarian blood supply. The accessory ovary originates from the separated portion of migrating ovarian primodium ${ }^{2,6,8}$.

The two proposed mechanisms for the supernumerary ovary are as follows

1. Arrested Gonocyte migration theory

2. Transplantation theory of germinal ridge Arrested gonocyte migration theory was proposed by Pearl \& Plotz in $1963^{10}$. They projected that some gonocytes can get arrested when they pass retroperitoneally along the dorsal mesentery and this failure in migration results in an additional ovary. The other is the transplantation theory of germinal ridge proposed by Printz et al, who suggested that following incorporation of the gonocytes, in the genital ridge, they are transplanted to another site ${ }^{13}$. The theory of ectopic implantation was anticipated by Kusaka and Mikuni in 2007 for ectopic ovarian tissue following surgery or inflammatory pathology ${ }^{5}$.

The supernumerary ovaries can be multiple and functional. They may be associated with other congenital anomalies of genitourinary system or found in association with other ovarian tumours. These associated conditions are also related to accessory ovary. Genital anomalies include accessory fallopian tubes, septate uterus, uterus 
didelphys, unicornuate uterus, etc and anomalies of urinary tract include unilateral renal agenesis, accessory suprarenal gland, agenesis of ureter and bladder diverticulum. Few of them may also show anomalies of gastrointestinal system like accessory pancreas or lobulated liver. In the present case, no such anomalies have been noted. However, the incidence of such anomalies is only $36 \%$. Literature have shown the association of a variety of ovarian tumours including Serous cystadenoma, mucinous cystadenoma, dermoid cyst, Brenner's tumour, fibroma, steroid cell tumour, etc with supernumerary ovary $1,4,7,9,11,14,15$. Collision tumour is defined as the coexistence of two different histologically distinct tumour entities in the same organ. It has been reported in various sites like lung, skin, gastrointestinal tract, central nervous system, adrenals, uterus, ovary, etc. Collision tumour is rare in ovary, of which teratoma is the most common accompanying component. In the present case, Mature cystic teratoma with thyroid component coexists with Serous cystadenoma.

When it is present in the right or left iliac fossa, it can mimic an appendicular mass, tubercular mass, peritoneal metastatic deposits or carcinoma colon. As a right iliac mass presenting with acute abdomen, a twisted supernumerary ovary needs to be differentiated from appendicular mass. They can also be confused for mesenteric cysts or lymphnodes. Pathological findings play an important role in distinguishing these conditions ${ }^{3}$. Malignancy in supernumerary ovaries are rarely reported and they are found to have no increased risk when compared to that of a normal ovary. But still, it needs careful follow up if not completely removed.

Supernumerary ovaries can be considered in the context of unidentifiable or idiopathic causes of abdominal pain, infertility, menorrhagia, dysmennorhea, hyperestrogenism, endometrial carcinoma or even ovarian hyper stimulation syndrome. This possibility is more common when there is associated Mullerian or urological anomaly $^{12}$.
Ultrasound is not diagnostic. MRI and laparoscopy are the necessary tools that aid in diagnosis and management of supernumerary ovaries. Further confirmation can be done by USG/ CT guided aspiration or biopsy. Frozen sections may also be helpful. Pathologist can even miss these supernumerary ovaries if adequate sampling is not done. This may lead to misdiagnosis or lower incidental reporting.

\section{Conclusion}

Supernumerary Ovary is a rare incidental finding noticed postoperatively and most of them goes unnoticed because of deficiency in reporting of such cases. Only less than 50 such cases have been reported so far. Still it is associated with other congenital anomalies and ovarian tumours. It should be brought to a limelight and one such case is presented here with another rare combination of collision tumour - serous cystadenoma and mature teratoma with thyroid component.

\section{Bibliography}

1. Barik S, Dhaliwal LK, Gopalan S, Rajwanshi A. Adenocarcinoma of the supernumerary ovary. 1991;34(1):75-77.

2. Cruikshank S. Supernumerary ovary: embryology. 1991;34(2):175-178.

3. Goel A. Differential diagnosis of a right iliac fossa mass | Radiology Reference Article | Radiopaedia.org. [accessed 2016 Nov

https://radiopaedia.org/articles/differentialdiagnosis-of-a-right-iliac-fossa-mass

4. Kuga T, Esato K, Takeda K, Sase M, Hoshii Y. A supernumerary ovary of the omentum with cystic change: Report of two cases and review of the literature. 1999;49(6):566-570.

5. Kusaka M, Mikuni M. Ectopic ovary: A case of autoamputated ovary with mature cystic teratoma into the cul-de-sac. 2007;33(3):368-370. 
6. LR W. Two cases of supernumerary ovary and one of accessory ovary, with an analysis of previously reported cases. 1959;78:1101-1119.

7. Mf L, Mm B. The ectopic ovary. A case report and review of the literature. 1991;115(3):233-235.

8. Moore KL, Persaud TVN, Torchia MG. The Developing Human: Clinically Oriented Embryology. Elsevier Health Sciences; 2015. 681 p.

9. Nomelini RS, Oliveira LJDC, Jammal MP, Adad SJ, Murta EFC. Serous papillary cystadenocarcinoma in supernumerary ovary. 2013;33(3):324.

10. Pearl M, Plotz EJ. Supernumerary ovary. Report of a case. 1963;21:253-256.

11. Peedicayil A, Sarada V, Jairaj P, Chandi SM. Ectopic Ovary in the Omentum. 1992;18(1):7-11.

12. Pithawa AK, Bansal AS, Kochar SPS. "Mesenteric cyst: A rare intra-abdominal tumour." 2014;70(1):79-82.

13. Printz JL, Choate JW, Townes PL, Harper RC. The embryology of supernumerary ovaries. 1973;41(2):246-252.

14. Rodríguez E, Pombo F, Alvarez C, Arnal F. Tumor in ectopic omental ovary in Mayer-Rokitansky-Küster-Hauser syndrome: CT findings. 1998;22(5):758759.

15. Zhigang Z, Wenlu S. An intrarenal supernumerary ovary concurrent with a completely duplicated pelvis and ureter. 2007;18(10):1243-1246. 